\title{
Photobiomodulation in Periodontology and Implant Dentistry: Part 2*
}

\author{
Leila Gholami, MSc, DDS, ${ }^{1}$ Sohrab Asefi, MSc, DDS, ${ }^{2}$ Amirarsalan Hooshyarfard, MSc, DDS, ${ }^{3}$ \\ Anton Sculean, PhD, DDS, ${ }^{4}$ Georgios E. Romanos, PhD, DDS, ${ }^{5}$ \\ Akira Aoki, PhD, DMD, ${ }^{6}$ and Reza Fekrazad, DDS, PhD, FLD, FICD ${ }^{7,8}$
}

\begin{abstract}
(Part 1 of this article can be located at www.liebertpub.com/doi/10.1089/photob.2019.4710.)

Objective: Finding evidence-based treatment strategies for low-level light therapy and the correct incorporation of these treatment methods in the clinical practice of periodontics.

Background: Photobiomodulation has been shown to have biostimulatory, anti-inflammatory, and analgesic effects that can be beneficial in periodontal and dental implant treatment procedures.

Methods: In this review, we have addressed some clinical questions regarding the potential clinical application of low-level light irradiation and its photobobiomodulatory effects in periodontology and implantology. The literature was searched for in vivo (animal or clinical) articles written in English in four electronic databases of PubMed, Scopus, Google Scholar, and Cochrane Library until April 2019. Only studies with low irradiation doses without any thermal effects used only for their photobiomodulatory purposes were included.

Results: We were able to find relevant studies for all of our questions, and positive effects for the application of light therapy were reported in most of the studies. However, there is still a great deal of heterogeneity in terms of study designs and most importantly in light irradiation devices and the parameters used. Due to this issue, it was not possible to reach specific evidence-based irradiation protocols for the questions addressed in this review.

Conclusions: Based on our search results, an obvious positive effect of low-level light therapy on stimulation of healing of periodontal soft and hard tissues and reduction of inflammation can be seen. Future well-designed randomized control studies with the same irradiation settings and systematic reviews evaluating the studies found on the questions mentioned are necessary to reach evidence-based recommendations.
\end{abstract}

Keywords: low-level light therapy, periodontology, dental implants

\section{Laser Photobiomodulation in Implant Dentistry}

\section{Clinical Question 1}

Does photobiomodulation promote bone formation around titanium implants?

Evidence search strategy. To FIND SUITABLE ARTICLES that have focused on the biostimulatory effect of photo- therapy on bone formation around dental implants a comprehensive electronic search was conducted by using key words of "bone formation," "Bone implant contact" "bone healing" OR" Dental implant" and the ones described earlier for low-level light therapy in the four data bases of PubMed, Google Scholar, Scopus, and Cochrane.

From the 97 articles chosen based on titles, 16 articles were finally included after evaluation of full texts. Any

\footnotetext{
${ }^{1}$ Dental Implants Research Center, Department of Periodontology, School of Dentistry, Hamadan University of Medical Sciences, Hamadan, Iran.

${ }^{2}$ Department of Orthodontics, School of Dentistry, Tehran University of Medical Sciences, Tehran, Iran.

${ }^{3}$ Dental Implants Research Center, Department of Periodontology, School of Dentistry, Hamadan University of Medical Sciences, Hamadan, Iran.

${ }^{4}$ Department of Periodontology, School of Dentistry, University of Bern, Bern, Switzerland.

${ }^{5}$ Department of Periodontology, School of Dental Medicine, Stony Brook University, Stony Brook, New York.

${ }^{6}$ Department of Periodontology, Graduate School of Medical and Dental Sciences, Tokyo Medical and Dental University, Tokyo, Japan.

${ }^{7}$ Radiation Sciences Research Center, Laser Research Center in Medical Sciences, AJA University of Medical Sciences, Tehran, Iran.

${ }^{8}$ International Network for Photo Medicine and Photo Dynamic Therapy (INPMPDT), Universal Scientific Education and Research Network (USERN), Tehran, Iran.

*Part 1 of this article can be located at www.liebertpub.com/doi/10.1089/photob.2019.4710.
} 
in vivo studies that had evaluated the effect of low-level light irradiation on bone formation around Titanium (Ti) surfaces were included.

Evidence-based recommendation and conclusion. There are many in vitro studies that have shown biostimulatory effects for low-intensity light therapy on cells and their osteogenic differentiation on Ti surfaces by measuring changes in different bone formation markers. ${ }^{1-3}$ Further studies have focused on testing this effect in animal or clinical settings to correctly translate these findings to clinical implication.

The majority of studies evaluating bone formation around Ti implants were conducted on animals, since it makes it possible to more clearly examine bone formation from a clinical and histological point of view. Most of these studies reported positive effects in terms of promotion of bone formation around Ti implants, and higher bone implant contacts (BICs) were reported (Table 1). ${ }^{4-19}$

Many researchers have confirmed that low-level light therapy can promote bone formation by stimulatory effects on osteoblasts, which can lead to greater osteo-integration of Ti implants. This has been evaluated in different study designs and laser irradiation parameters.

In a study in sheep, Jakse et al. found no significant regenerative effect after sinus lift procedure with autologous graft (iliac crest) under low-level laser (LLL) irradiation; however, they also considered it to be possibly effective in osseointegration of dental implants inserted after sinus augmentation. ${ }^{9}$ Another animal study was also conducted by Soares et al. on implants integration in auto and xenografts in rabbits. ${ }^{13}$ They observed an increased bone formation in the bone-implants interface after LLL irradiation. However, it was noted that bone formation was significantly increased in autografts but was not statistically significant with xenografts. The experimental animals (sheep and rabbit) used, laser wavelength (680 or $780 \mathrm{~nm}$ ), and output energy densities (3-4 or $16-21 \mathrm{~J} / \mathrm{cm}^{2}$ ) were different between these two studies, which might have led to the different results obtained.

Lopes et al. have also showed some positive effects for adjunctive photo therapy. In their study, calcium-hydroxyapatite absorption and implant integration were significantly increased by application of LLL irradiation. ${ }^{10}$

Pereira et al. have irradiated Ti implants inserted in tibia of rabbits with low-intensity laser with a 48-h interval for 14 days. According to their results with laser therapy, BIC had a significant increase at 3-6 weeks evaluation times. However, it did not affect the area of bone formed within the threads. ${ }^{11}$ In 2004 , Khadra et al. reported many positive effects of LLL irradiation. According to their findings, irradiated implants had a greater bond to bone based on tensile pullout test results. A greater amount of calcium and phosphorus content on the irradiated implant surfaces were observed compared with the nonirradiated group, and bone maturation was also faster in irradiated bone. ${ }^{6}$

In another animal study by Kim et al., expression of osteoprotegrin (OPG), receptor activator of nuclear factor kappa-B ligand (RANKL), and RANK was shown to be influenced by low-level laser irradiation and an increase of metabolic bone activity and bone tissue cell activity was observed. $^{8}$

In an interesting research by de Vasconcellos et al. in 2016 in rats, low levels of infrared diode laser were found to be able to improve the initial phase of bone formation and the osseointegration process of $\mathrm{Ti}$ scaffolds in osteopenic and normal rats. ${ }^{17}$

In a recent study, Mikhail et al. have evaluated the radiodensitometric changes in bone density along the boneimplant interface. They observed that the rate of increase and mean differences was significantly higher in the laser group. ${ }^{19}$ Their conclusion was that low-intensity laser irradiation significantly promoted bone healing and increased the speed of the osseointegration process.

Overall, it seems that energy densities of around 10$20 \mathrm{~J} / \mathrm{cm}^{2}$ performed every other day for 2 weeks may be considered an effective treatment procedure in terms of promoting bone formation around Ti implants. Although there is once again great variation observed in the irradiation protocols used in the current available studies, further research with similar and improved study designs will still be valuable.

\section{Clinical Question 2}

\section{Can photobiomodulation influence Ti implant stability?}

Evidence search strategy. Once again, the literature was searched by using key words of "dental implant stability," "osseointegration," "stability" and key words used for low-level light therapy in the four electronic databases previously mentioned. Forty-nine abstracts were chosen and after full text evaluation, 16 in vivo articles that met the selection criteria for this review question were included. Any animal or human studies evaluating Ti, implant stability, or osseointegration with the adjunctive use of lowintensity light were considered eligible. There were also a few reports on mini-implants or miniscrews used for temporary purposes that were excluded here and are discussed separately in the following question.

Evidence-based recommendations and conclusion. Decreasing the duration of osseointegration has been a topic of interest for laser implant research for many years. Since it is proposed that low-level light therapy can influence osteoblast cells and the osseointegration process, it seems possible that it can also be effective at increasing the stability of Ti implants. We were able to find 16 articles until April 2019 focusing on this subject. Seven were clinical studies performed on human subjects and 9 were animal studies. In these studies, implant stability was measured by using different methods such as removal torque tests, tensile pullout test, resonance frequency analysis (RFA) devices, or measuring implant stability quotient (ISQ). The animal studies have all reported that photo therapy with low levels of light can provide greater bone-implant interface strength and also improve the osseointegration process (Table 2). ${ }^{6,15,18,20-33}$ Removal torque values increased significantly along with time even after a single laser irradiation compared with a nonirradiated control group in the study by Boldrini et al. ${ }^{24}$ Gomes et al. have interestingly shown that higher energy levels $\left(10\right.$ or $20 \mathrm{~J} / \mathrm{cm}^{2}$ ) promote more implant-bone contact compared with a lower energy $\left(5 \mathrm{~J} / \mathrm{cm}^{2}\right)$. Better ISQ values were also observed in the $20 \mathrm{~J} / \mathrm{cm}^{2}$ group (this article was published in the Quintessense journal in the same year with the Mayer et al. bibliography). ${ }^{15,26}$

In the human studies, implant stability values (ISQ measurements) were shown to be increased in low-level 


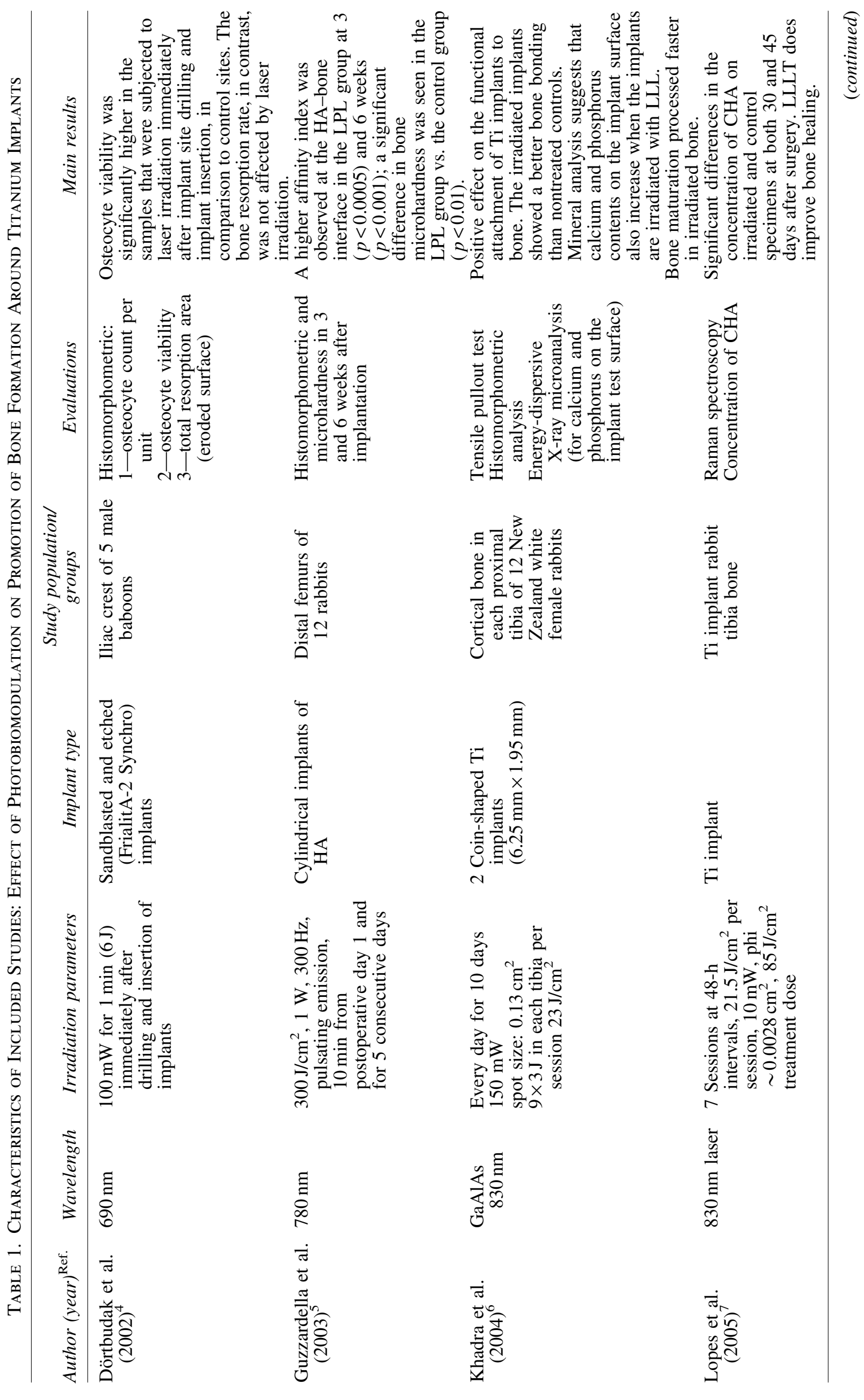




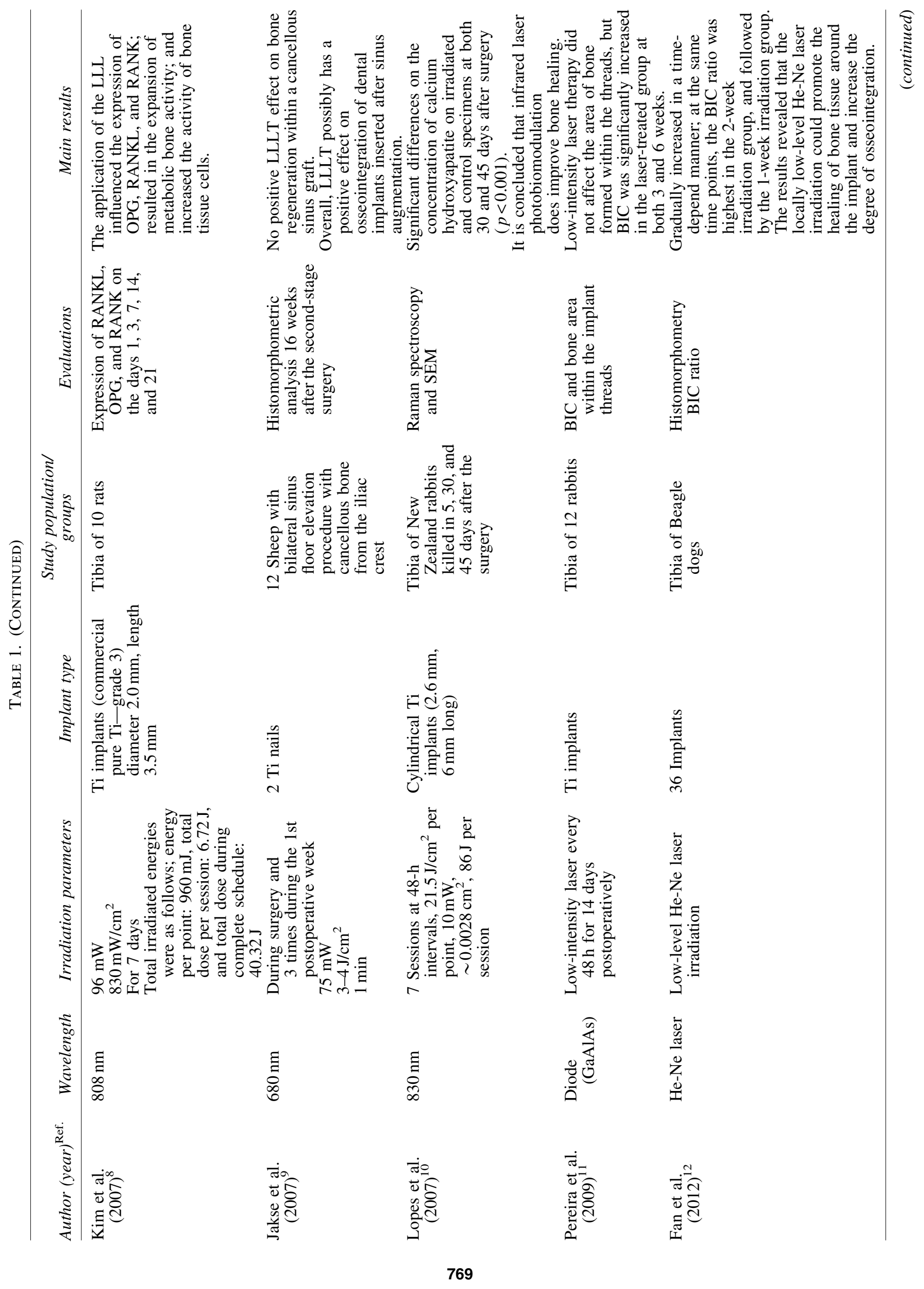




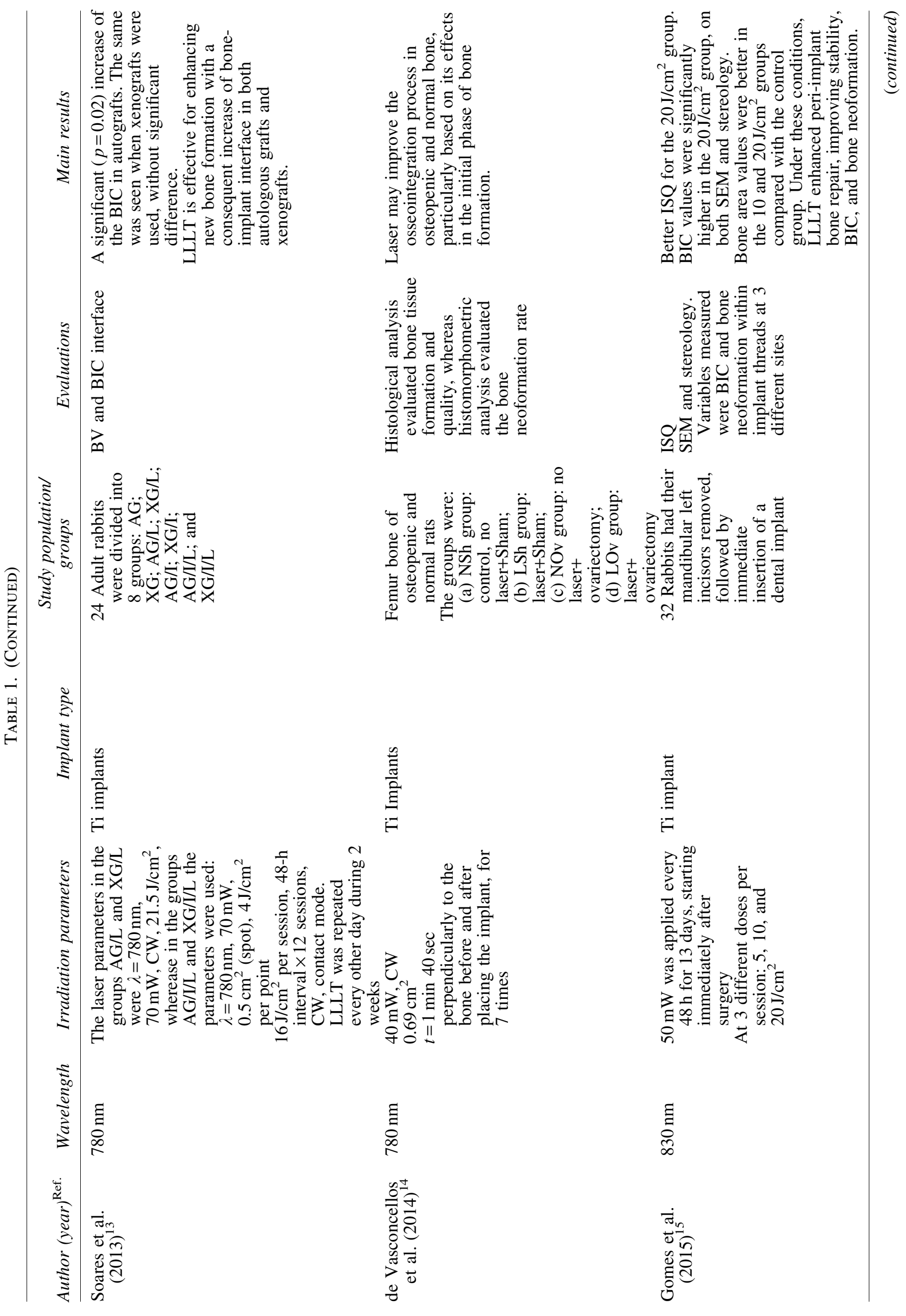




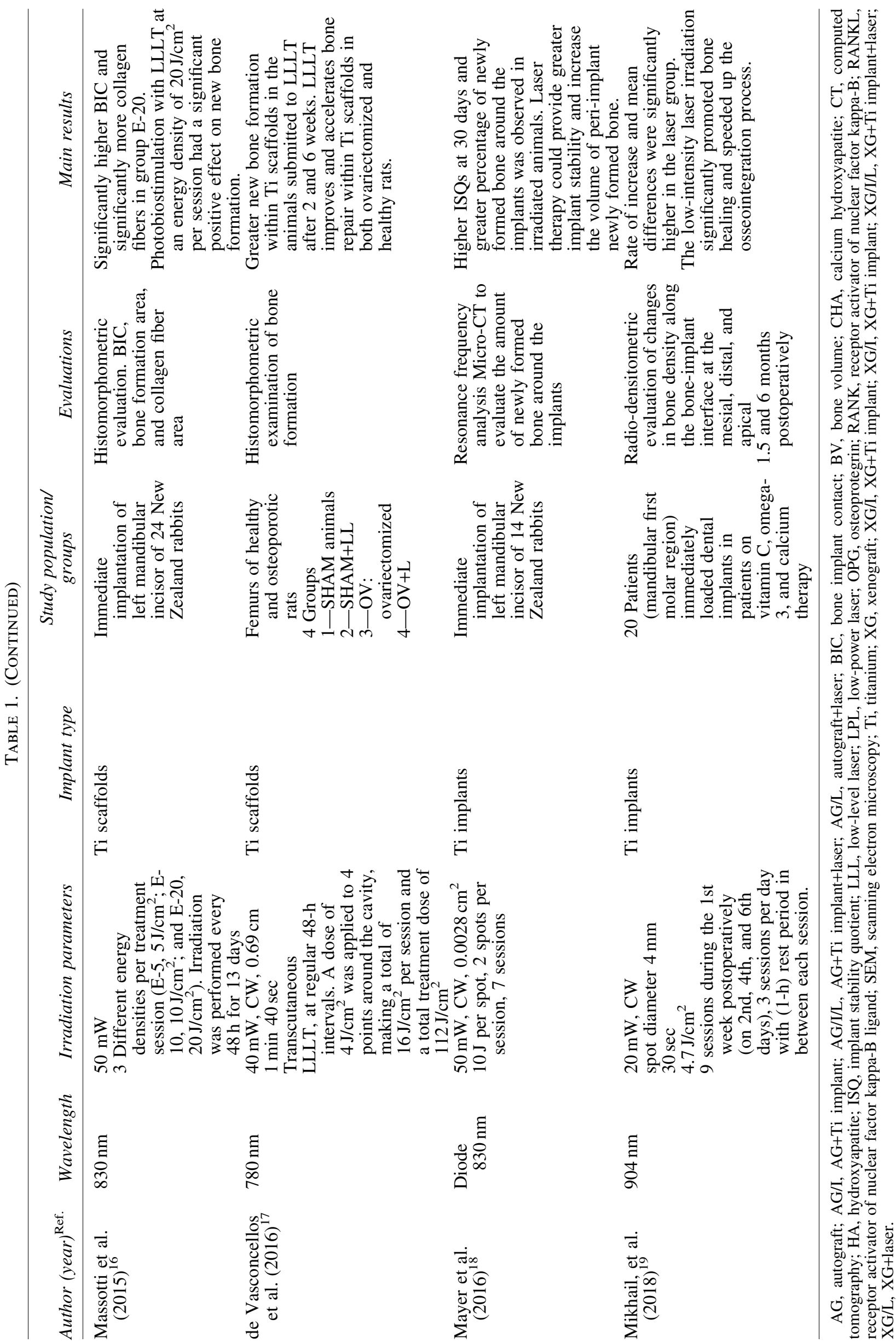




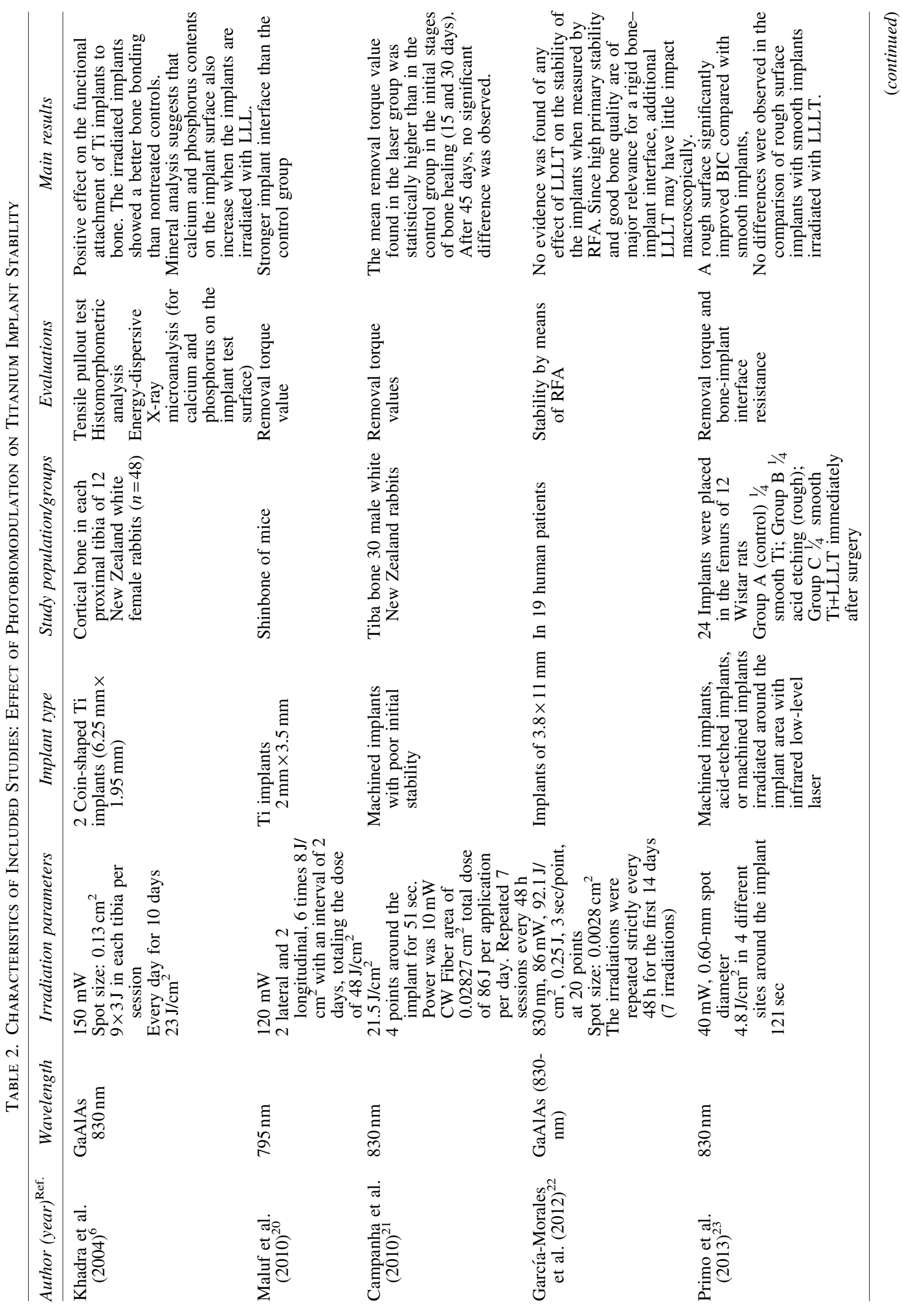




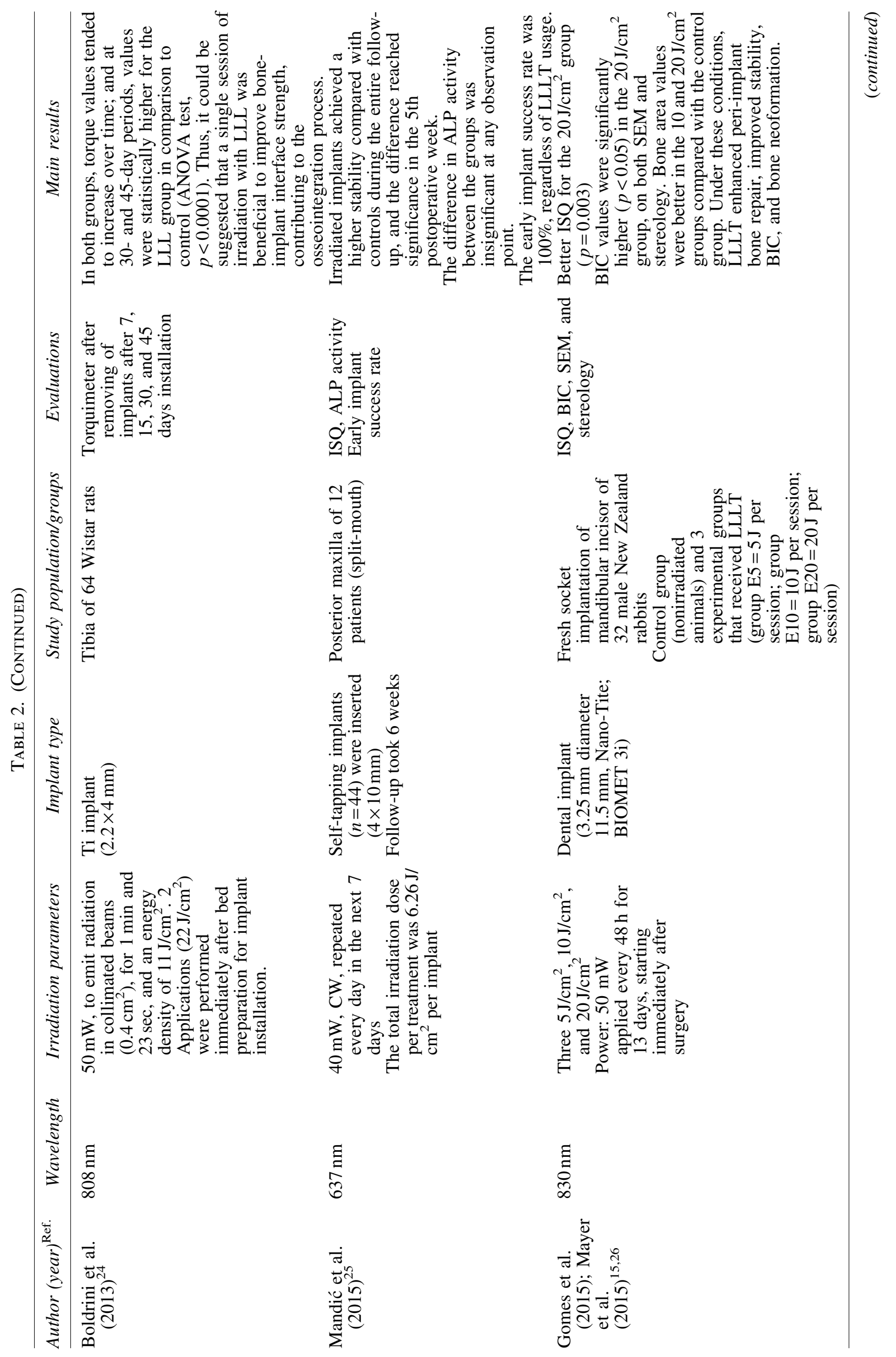




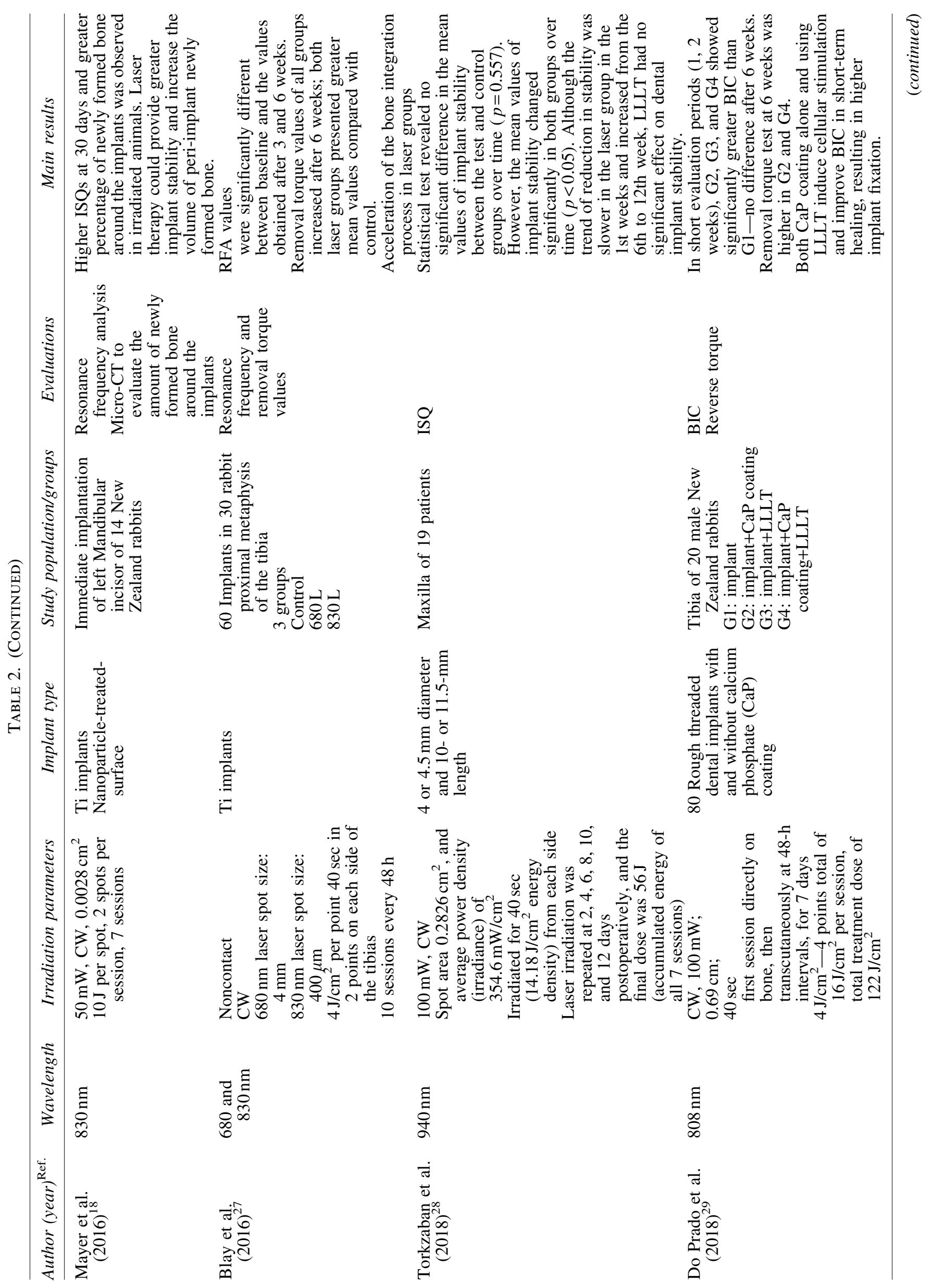




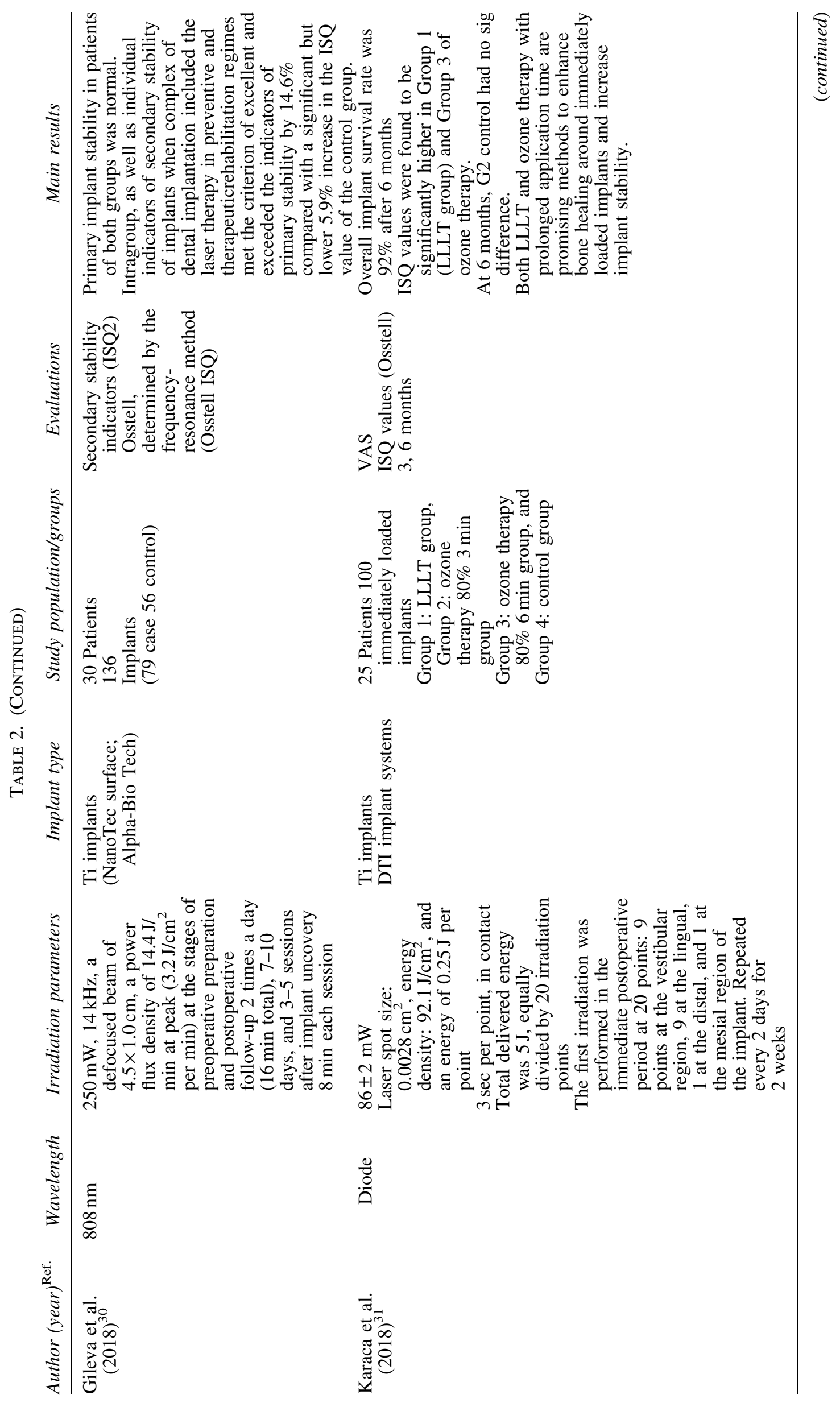




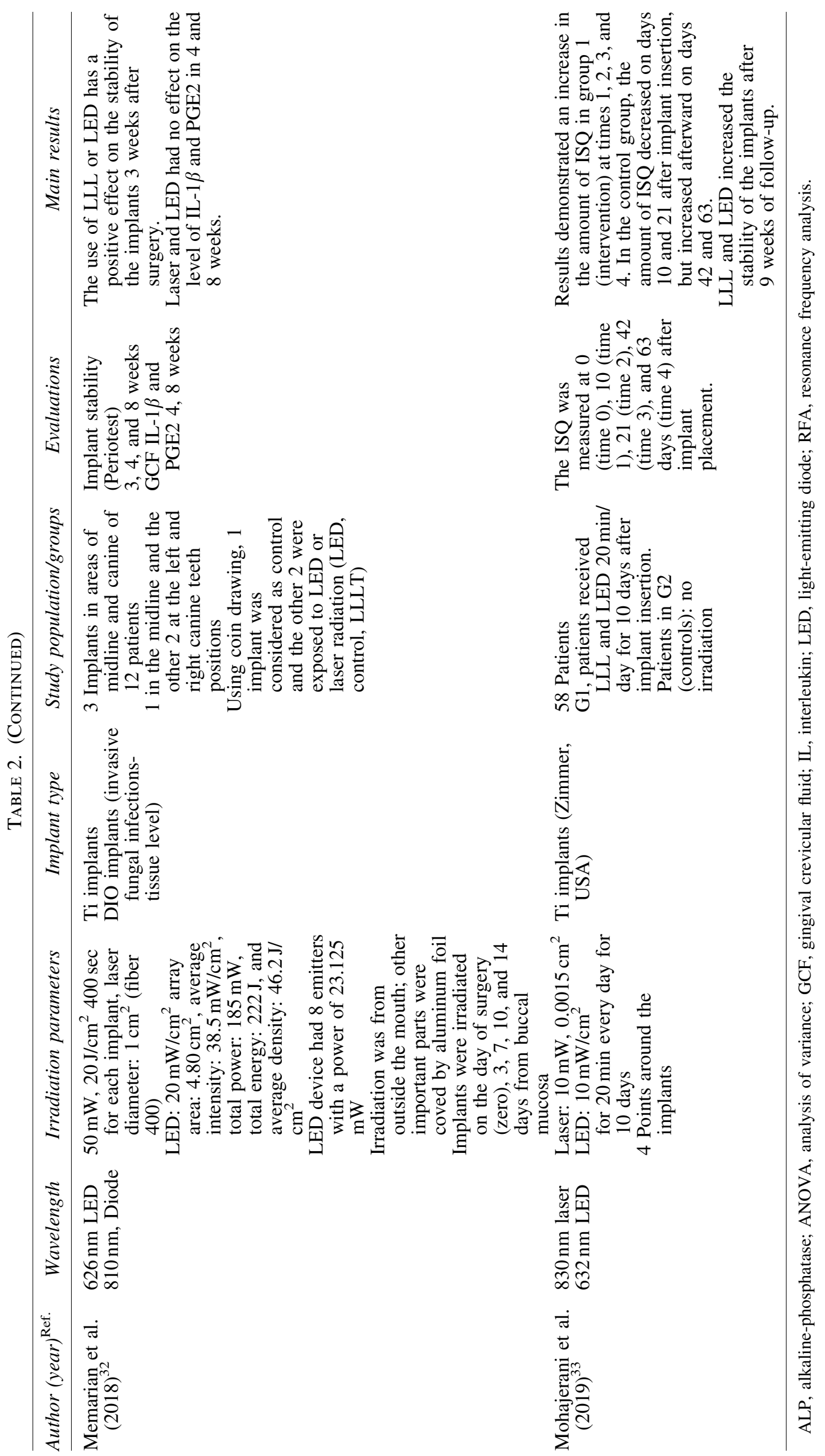


laser therapy (LLLT) groups. ${ }^{25,30,31,33}$ However, Torkzaban et al. found no significant effect of adjunctive 940-nm low laser therapy on implant stability. In another randomized clinical trial, García-Morales (2012) reported that LLL was not able to significantly increase implant stability when assessed by resonance frequency analysis (RFA). ${ }^{22,28}$

Implant surface roughness is one of the factors that may also influence osseointegration and stability. In the animal study by Primo et al., three groups of smooth Ti implants, rough surface (acid etched) implants and smooth implants irradiated with LLLT were compared. The BIC of rough surface implants was greater than with smooth implants. However, there was no statistically significant difference between rough surface implants and smooth implants irradiated with LLLT.

Overall, the results should be interpreted with caution since many factors such as implant types, various irradiation parameters, and implant stability measurement methods had a wide variation in the studies and may possibly influence the results obtained. Further well-designed studies can help better elucidate this potential application of photo biomodulation in implant dentistry.

\section{Clinical Question 3}

Does photobiomodulation irradiation influence mini-Ti implant (un osteo- integrated miniscrew) stability and success?

Evidence search strategy. To find relevant research focusing on adjunctive low-level light therapy used for improving non osteo-integrated miniscrew or mini-implant stability or success, PubMed, Google Scholar, Scopus, and Cochrane databases were searched by using the key words we had selected for photobiomodulation and key words of "mini implant" OR "mini screw" AND "stability" OR "success." In vivo animal or human studies evaluating the stability and success of mini-implants or miniscrews were considered eligible. Eight studies were identified based on our inclusion criteria (Table 3). ${ }^{34-39}$

Evidence-based conclusion and recommendation. Improving orthodontic anchorage can be achieved by placing mini-implants or miniscrews in the alveolar bone. Enhancing the stability and therefore the success of these implants by low-level light photo biomodulation has attracted the attention of many researchers in this field in recent years. We were able to find five animal studies and three studies on human subjects. In most of the animal studies, it has been concluded that LLLT may be useful in enhancement of the stability of mini-implants based on measuring implant stability with different methods. ${ }^{34-37,40}$ Interestingly, in the Uysal et al. study, a significant increase was found in ISQ values when photobiomodulation therapy was used on miniscrews in all three different force groups $(0,150$, and $300 \mathrm{cN}$ ). However, as force levels increased, ISQ values decreased in non-irradiated control miniscrews. ${ }^{35}$ In a histological study by Garcez et al. in the irradiated group, the number of inflammatory cells was less than the control group and a more intense new bone formation was observed around the irradiated miniscrews. ${ }^{37}$

The results of the three clinical studies have also shown improved stability and reduced inflammation around min- iscrews or implants placed in human subjects. However, the improved stability results (measured by Periotest) reported in Osman et al.'s study were not statistically significant. ${ }^{38,39,41}$ They have also evaluated the inflammatory markers in the peri-implant crevicular fluid (PGF) and concluded that since LLLT modulates the initial inflammation after the insertion of mini-implants, it seems that it is possible to increase the mini-implant success prognosis and decrease patient discomfort with adjunctive photobiomodulation.

The evidence seems to be in favor of the use of adjunctive photobiomodulation with mini-implant/miniscrew placement to improve their success and stability. To reach better evidence-based conclusions, future randomized clinical trials studies with similar study designs seem to be necessary.

\section{Clinical Question 4}

\section{Does photobiomodulation influence proliferation} and viability of non-osseous periodontal cells around Ti implants?

Evidence search strategy. The terms "cells" OR "gingival fibroblast" OR "periodontal ligament" AND "titanium" were used in conjunction with the keywords previously mentioned for photobiomolulation to find studies on proliferation and viability of non-osseous cells on Ti surfaces after LLLT. After title screening, 25 articles remained, out of which only two studies matched our criteria for this question and they were in vitro studies conducted on nonosseous cells (Table 4). ${ }^{42,43}$

Evidence-based conclusion and recommendation. The effect of low-level light therapy on osteogenic cells has been well studied and there are a great number of studies on this topic. However, we were only able to find two studies by Khadra that had evaluated the effect of 830-nm low-level laser irradiation on pure Ti disks and the response of nonosseous cells (fibroblast cells). The results showed that laser irradiation significantly improved cell attachment, colonyforming efficiency, and clonal growth rates. However, cell viability did not exhibit a significant difference between the laser and control groups. ${ }^{42,43}$

In the second study, different doses of laser irradiation were evaluated. Both single and multiple doses of LLLT were able to enhance the attachment of cells. There was an agreement in the proliferation assay and staining and enzyme-linked immunosorbent assay indicating significantly better results for the group receiving 1.5 and $3 \mathrm{~J} / \mathrm{cm}^{2}$ laser therapy after $72 \mathrm{~h}$ and 7 days. ${ }^{43}$

It may be concluded that according to these in vitro studies, the attachment and proliferation of human gingival fibroblasts on Ti may be enhanced by LLLT in a dosedependent manner and could be beneficial in clinical practice.

\section{Future Recommendations}

Laser technology has definitely brought dentistry into a new era. Many new applications based on the fascinating effect of light on oral and dental tissues has become the topic of a great and growing number of researches conducted in this field. 


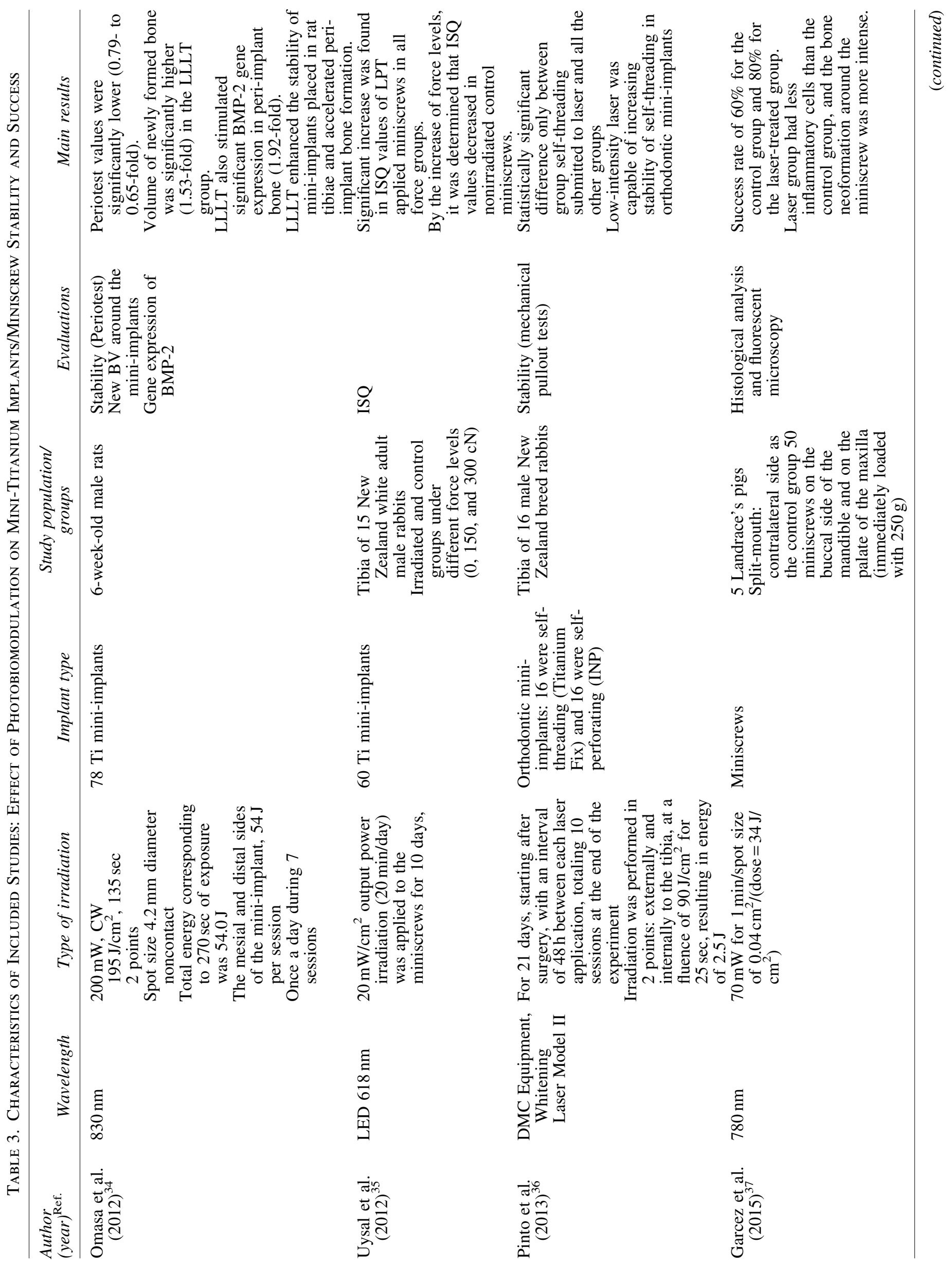




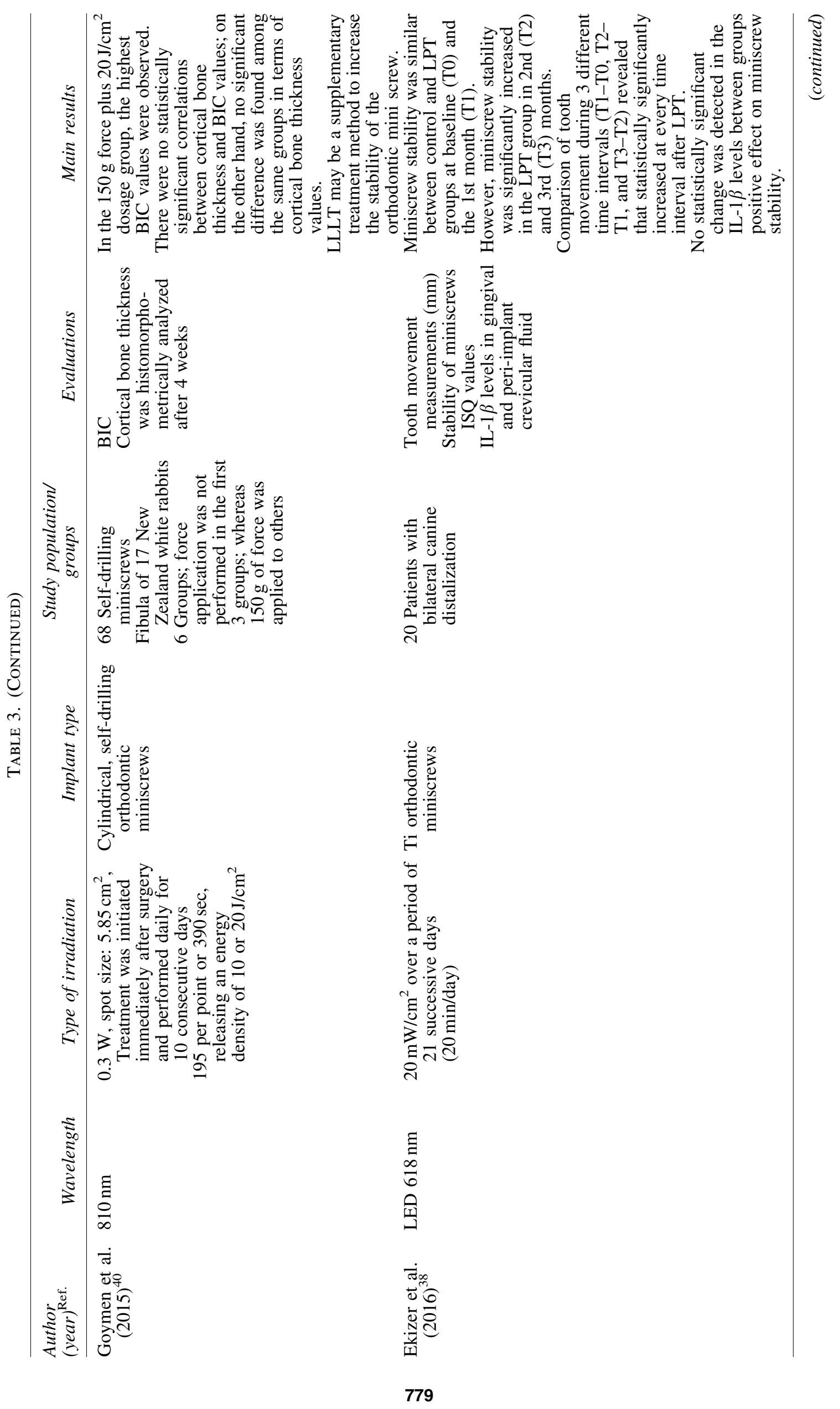




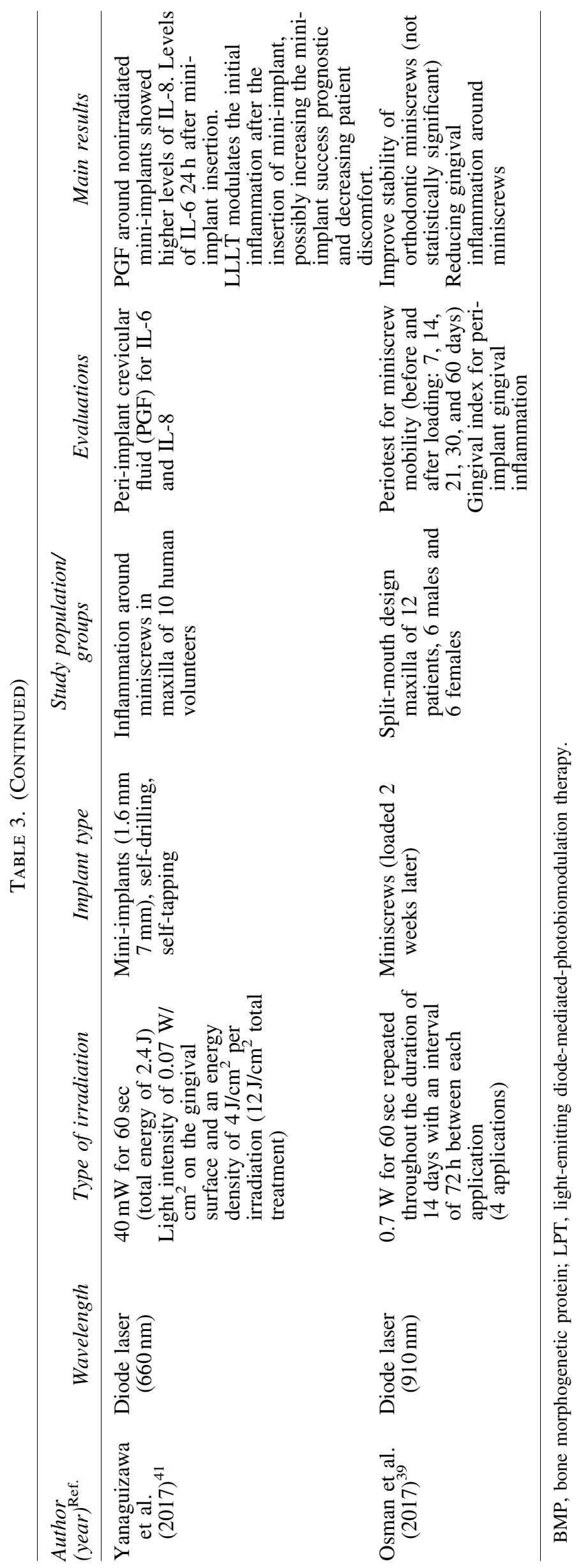




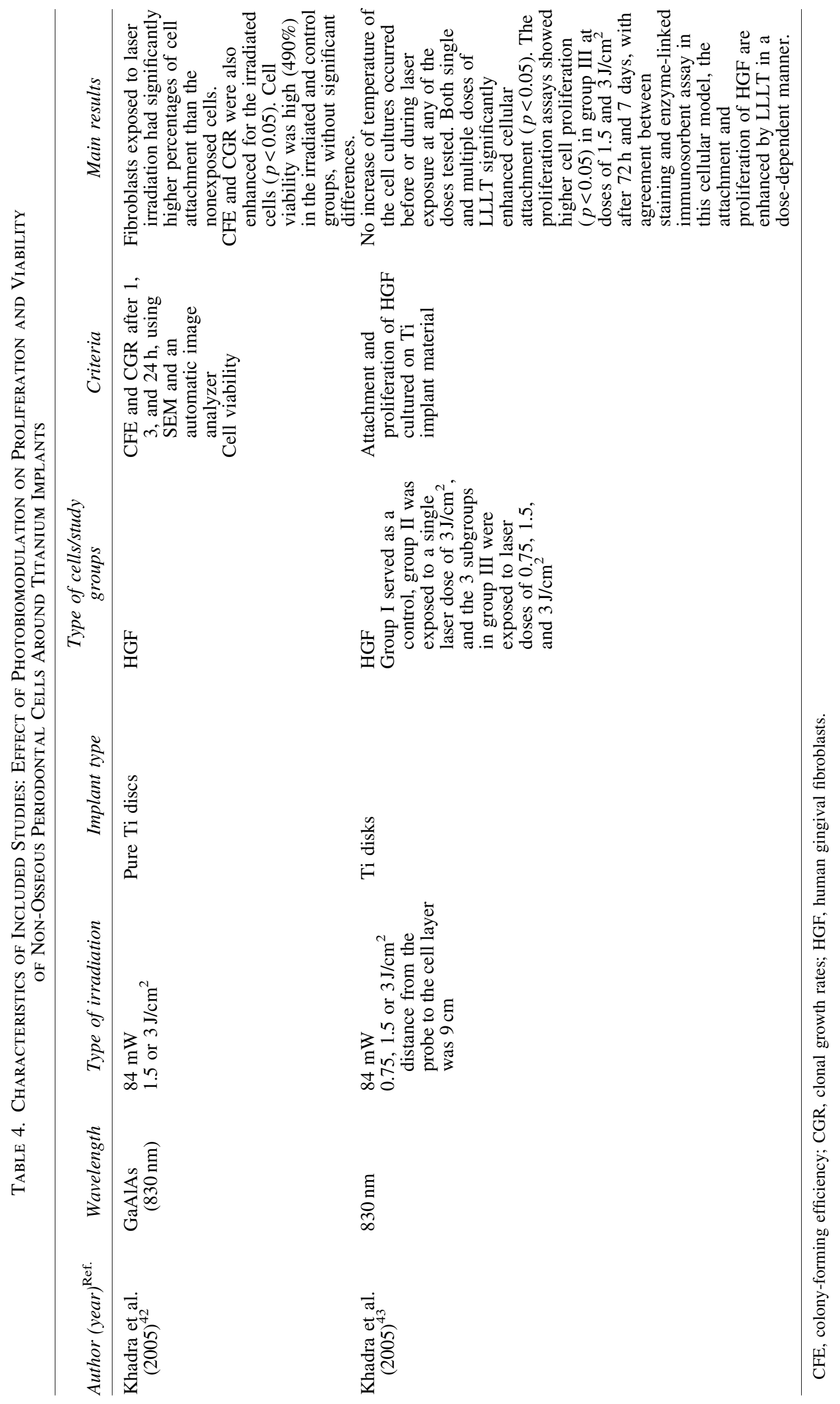


Photobiomodulation is a sensitive procedure and small changes in irradiation or tissue-related factors may lead to major changes in the results and outcomes. Thus, finding out the appropriate settings for reaching favorable and practical results for clinical applications needs to be determined by conducting well-designed in vitro and in vivo studies. Although there are a lot of potential applications for this kind of photo therapy in periodontology and implant dentistry and many researchers have reported positive effects, however, we are still not able to reach evidence-based conclusions for many of its applications or recommend a specific treatment protocol. This is mainly due to the great variation observed in the irradiation parameters used and different study designs of the available literature.

Effective comparison of clinical studies necessitates designing precise randomized clinical trials with longer follow-up periods and similar inclusion criteria for periodontal patients and situations. Since periodontal disease is multifactorial in nature, confounding factors that might affect the progression of disease should also be carefully considered in study designs. Other methodological factors such as sample size calculations, allocation, randomization, and blinding methods should also be considered to reduce the risk of bias of studies. Finally, as mentioned in many systematic reviews and studies in this field, finding the optimal irradiation parameters to reach favorable inhibitory or biostimulatory effects is of great importance; therefore, it is recommended that laser parameters should now be selected based on the current available evidence and comprehensively reported in a standardized manner. In addition, different laser settings need to be comparatively evaluated to reach suitable parameters and evidenced-based protocols and guidelines for clinical application covered within Part $1 .^{44}$

\section{Author Disclosure Statement}

No competing financial interests exist.

\section{Funding Information}

The authors received no financial support for the research.

\section{References}

1. Grassi FR, Ciccolella F, D'Apolito G, et al. Effect of lowlevel laser irradiation on osteoblast proliferation and bone formation. J Biol Regul Homeost Agents 2011;25:603614.

2. Khadra M. The effect of low level laser irradiation on implant-tissue interaction. In vivo and in vitro studies. Swed Dent J Suppl 2005:1-63.

3. Petri AD, Teixeira LN, Crippa GE, Beloti MM, de Oliveira PT, Rosa AL. Effects of low-level laser therapy on human osteoblastic cells grown on titanium. Braz Dent J 2010;21: 491-498.

4. Dörtbudak O, Haas R, Mailath-Pokorny G. Effect of lowpower laser irradiation on bony implant sites. Clin Oral Implants Res 2002;13:288-292.

5. Guzzardella GA, Torricelli P, Nicoli-Aldini N, Giardino R. Osseointegration of endosseous ceramic implants after postoperative low-power laser stimulation: an in vivo comparative study. Clin Oral Implants Res 2003;14:226232 .
6. Khadra M, Ronold HJ, Lyngstadaas SP, Ellingsen JE, Haanaes HR. Low-level laser therapy stimulates boneimplant interaction: an experimental study in rabbits. Clin Oral Implants Res 2004;15:325-332.

7. Lopes CB, Pinheiro AL, Sathaiah S, Duarte J, Cristinamartins M. Infrared laser light reduces loading time of dental implants: a Raman spectroscopic study. Photomed Laser Surg 2005;23:27-31.

8. Kim YD, Kim SS, Hwang DS, et al. Effect of low level laser treatment after installation of dental titanium implantimmunohistochemical study of vascular endothelial growth factor: an experimental study in rats. Laser Phys Lett 2007; 4:681-685.

9. Jakse N, Payer M, Tangl S, Berghold A, Kirmeier R, Lorenzoni M. Influence of low-level laser treatment on bone regeneration and osseointegration of dental implants following sinus augmentation. An experimental study on sheep. Clin Oral Implants Res 2007;18:517-524.

10. Lopes CB, Pinheiro AL, Sathaiah S, Da Silva NS, Salgado MA. Infrared laser photobiomodulation (lambda $830 \mathrm{~nm}$ ) on bone tissue around dental implants: a Raman spectroscopy and scanning electronic microscopy study in rabbits. Photomed Laser Surg 2007;25:96-101.

11. Pereira CL, Sallum EA, Nociti Jr FH, Moreira RW. The effect of low-intensity laser therapy on bone healing around titanium implants: a histometric study in rabbits. Int J Oral Maxillofac Implants 2009;24:47-51.

12. Fan Q, Xu St, Liu Jg, Cong L. Effect of low-level laser on the healing of bone tissues around the implant in tibia of Beagle dogs. Chin J Tissue Eng Res 2012;16:5581-5585.

13. Soares LG, Magalhaes EB, Magalhaes CA, Ferreira CF, Marques AM, Pinheiro AL. New bone formation around implants inserted on autologous and xenografts irradiated or not with IR laser light: a histomorphometric study in rabbits. Braz Dent J 2013;24:218-223.

14. de Vasconcellos LM, Barbara MA, Deco CP, et al. Healing of normal and osteopenic bone with titanium implant and low-level laser therapy (GaAlAs): a histomorphometric study in rats. Lasers Med Sci 2014;29:575-580.

15. Gomes FV, Mayer L, Massotti FP, et al. Low-level laser therapy improves peri-implant bone formation: resonance frequency, electron microscopy, and stereology findings in a rabbit model. Int J Oral Maxillofac Surg 2015;44: 245-251.

16. Massotti FP, Gomes FV, Mayer L, et al. Histomorphometric assessment of the influence of low-level laser therapy on peri-implant tissue healing in the rabbit mandible. Photomed Laser Surg 2015;33:123-128.

17. de Vasconcellos LM, Barbara MA, Rovai ES, et al. Titanium scaffold osteogenesis in healthy and osteoporotic rats is improved by the use of low-level laser therapy (GaAlAs). Lasers Med Sci 2016;31:899-905.

18. Mayer L, Gomes FV, de Oliveira MG, de Moraes JF, Carlsson L. Peri-implant osseointegration after low-level laser therapy: micro-computed tomography and resonance frequency analysis in an animal model. Lasers Med Sci 2016;31:1789-1795.

19. Mikhail FF, El-Din M, Ibrahim T, Zekry K, Nemat A, Nasry S. Effect of laser therapy on the osseointegration of immediately loaded dental implants in patients under vitamin C, omega-3 and calcium therapy. Open Access Maced J Med Sci 2018;6:1468-1474.

20. Maluf AP, Maluf RP, Da Rocha Brito C, França FMG, De Brito Jr RB. Mechanical evaluation of the influence of low- 
level laser therapy in secondary stability of implants in mice shinbones. Lasers Med Sci 2010;25:693-698.

21. Campanha BP, Gallina C, Geremia T, et al. Low-level laser therapy for implants without initial stability. Photomed Laser Surg 2010;28:365-369.

22. García-Morales JM, Tortamano-Neto P, Todescan FF, de Andrade Jr JC, Marotti J, Zezell DM. Stability of dental implants after irradiation with an 830-nm low-level laser: a double-blind randomized clinical study. Lasers Med Sci 2012;27:703-711.

23. Primo BT, da Silva RC, Grossmann E, Miguens Jr SA, Hernandez PA, Silva Jr AN. Effect of surface roughness and low-level laser therapy on removal torque of implants placed in rat femurs. J Oral Implantol 2013;39:533-538.

24. Boldrini C, de Almeida JM, Fernandes LA, et al. Biomechanical effect of one session of low-level laser on the bone-titanium implant interface. Lasers Med Sci 2013;28: 349-352.

25. Mandić B, Lazić Z, Marković A, et al. Influence of postoperative low-level laser therapy on the osseointegration of self-tapping implants in the posterior maxilla: a 6-week split-mouth clinical study. Vojnosanit Pregl 2015;72: 233-240.

26. Mayer L, Gomes FV, Carlsson L, Gerhardt-Oliveira M. Histologic and resonance frequency analysis of periimplant bone healing after low-level laser therapy: an in vivo study. Int $\mathbf{J}$ Oral Maxillofac Implants 2015;30: 1028-1035.

27. Blay A, Blay CC, Tunchel S, et al. Effects of a lowintensity laser on dental implant osseointegration: removal torque and resonance frequency analysis in rabbits. J Oral Implantol 2016;42:316-320.

28. Torkzaban P, Kasraei S, Torabi S, Farhadian M. Low-level laser therapy with $940 \mathrm{~nm}$ diode laser on stability of dental implants: a randomized controlled clinical trial. Lasers Med Sci 2018;33:287-293.

29. Do Prado RF, El Abras Ankha MDV, Bueno DAG, et al. Cap coating and low-level laser therapy to stimulate early bone formation and improve fixation of rough threaded implants. Implant Dent 2018;27:660-666.

30. Gileva OS, Chuprakov MA, Libik TV, Syutkina ES, Mirsaeva FZ. Dynamics of dental implant stability indicators (ISQ) using low-level laser therapy in treatment and prevention modes. Russ J Biomech 2018;22:450-462.

31. Karaca I, Ergun G, Ozturk D. Is Low-level laser therapy and gaseous ozone application effective on osseointegration of immediately loaded implants? Niger J Clin Pract 2018; 21:703-710.

32. Memarian J, Ketabi M, Amini S. The effect of low-level laser $810 \mathrm{~nm}$ and light-emitting diode photobiomodulation $(626 \mathrm{~nm})$ on the stability of the implant and inflammatory markers interleukin-1 beta and prostaglandin E2, around implants. Dent Res J 2018;15:283-288.

33. Mohajerani H, Salehi AM, Tabeie F, Shafiei S, Tabrizi R. Can low-level laser and light-emitting diode enhance the stability of dental implants? J Maxillofacial Oral Surg 2019;1-5.

34. Omasa S, Motoyoshi M, Arai Y, Ejima K, Shimizu N. Low-level laser therapy enhances the stability of orthodontic mini-implants via bone formation related to BMP-2 expression in a rat model. Photomed Laser Surg 2012;30: 255-261.
35. Uysal T, Ekizer A, Akcay H, Etoz O, Guray E. Resonance frequency analysis of orthodontic miniscrews subjected to light-emitting diode photobiomodulation therapy. Eur $\mathrm{J}$ Orthod 2012;34:44-51.

36. Rodrigues Pinto M, Dos Santos RL, Pithon MM, De Souza Araújo MT, Braga JPV, Nojima LI. Influence of lowintensity laser therapy on the stability of orthodontic miniimplants: a study in rabbits. Oral Surg Oral Med Oral Pathol Oral Radiol 2013;115:e26-e30.

37. Garcez AS, Suzuki SS, Martinez EF, Iemini MG, Suzuki H. Effects of low-intensity laser therapy over mini-implants success rate in pigs. Lasers Med Sci 2015;30:727-732.

38. Ekizer A, Turker G, Uysal T, Guray E, Tasdemir Z. Light emitting diode mediated photobiomodulation therapy improves orthodontic tooth movement and miniscrew stability: a randomized controlled clinical trial. Lasers Surg Med 2016;48:936-943.

39. Osman A, Abdel Moneim A, El Harouni N, Shokry M. Long-term evaluation of the effect of low-level laser therapy on orthodontic miniscrew stability and peri-implant gingival condition: a randomized clinical trial. J World Fed Orthodontists 2017;6:109-114.

40. Goymen M, Isman E, Taner L, Kurkcu M. Histomorphometric evaluation of the effects of various diode lasers and force levels on orthodontic mini screw stability. Photomed Laser Surg 2015;33:29-34.

41. Yanaguizawa MS, Suzuki SS, Martinez EF, Suzuki H, Pelegrin MCJ, Garcez AS. Effects of low-level laser therapy in orthodontic patients on immediate inflammatory response after mini-implants insertion: a preliminary report. Photomed Laser Surg 2017;35:57-63.

42. Khadra M, Kasem N, Lyngstadaas SP, Haanæs HR, Mustafa K. Laser therapy accelerates initial attachment and subsequent behaviour of human oral fibroblasts cultured on titanium implant materia: a scanning electron microscopic and histomorphometric analysis. Clin Oral Implants Res 2005;16:168-175.

43. Khadra M, Lyngstadaas SP, Haanaes HR, Mustafa K. Determining optimal dose of laser therapy for attachment and proliferation of human oral fibroblasts cultured on titanium implant material. J Biomed Mater Res A 2005;73: 55-62.

44. Gholami L, Asefi S, Hoshyarfard A, et al. Photobiomodulation in periodontology and implant dentistry-Part 1. Photobiomodulation Photomed Laser Surg 2019;37:739_ 764. DOI: $10.1089 /$ pho.2019.4710.

Address correspondence to:

Reza Fekrazad, DDS, PhD, FLD, FICD

Radiation Sciences Research Center Laser Research Center in Medical Sciences AJA University of Medical Sciences

Flat No 12, Mooj Building

First Behestan, Pasdaran Street Tehran 1946883893

Iran

E-mail: rezafekrazad@gmail.com

Received: July 26, 2019

Accepted after revision: July 26, 2019

Published online: November 20, 2019.

Part 1 of this article can be located at www.liebertpub.com/doi/10.1089/photob.2019.4710. 